\title{
EDITORIAL AND COMMENT \\ Can Parsimonious Practice Please Patients and Practitioners? The Case of Spine Imaging
}

\author{
Richard A. Deyo, MD, MPH \\ 'Department of Family Medicine, Department Of Medicine, Department Of Public Health and Preventive Medicine, Oregon Health \& Science \\ University, Portland, OR, USA; ${ }^{T}$ The Oregon Institute for Occupational Health Sciences, Oregon Health \& Science University, Portland, OR, USA; ${ }^{3}$, \\ Portland, OR, USA.
}

J Gen Intern Med 31(2):140-1

DOI: $10.1007 / \mathrm{s} 11606-015-3523-Z$

(C) Society of General Internal Medicine 2015

$\mathrm{T}$ here now seems to be broad agreement that lumbar spine imaging is an overused clinical procedure. Clinical guidelines recommend against routine lumbar spine imaging for patients with nonspecific low back pain, ${ }^{1}$ and such imaging has become a high-priority target for the Choosing Wisely campaign. Nonetheless, spine imaging rates are generally higher than expected. Tan and colleagues now provide evidence that high use continues, and that there is wide variation among individual physicians in the use of imaging for older patients. $^{2}$

Part of the problem with spine imaging is that serious underlying conditions are rare, while anatomical "abnormalities" are extremely common, and their prevalence increases with age. For example, disc degeneration and bulging discs are found in more than $50 \%$ of pain-free adults aged $40-49$, and among those in their 60 s, nearly $70 \%$ or more have disc degeneration, disc space narrowing, and disc bulges. ${ }^{3}$ Such "incidentalomas" may alarm both doctors and patients, and lead to ill-advised interventions.

Indeed, there is growing evidence that unnecessary spine imaging may lead to a cascade of subsequent diagnostic and treatment interventions that do not improve outcomes, and may increase risk. Spine imaging is one of the few examples of a diagnostic test (rather than a therapeutic intervention) for which we have randomized trials to assess the impact. For low-risk patients, a meta-analysis of randomized controlled trials (RCTs) has suggested that imaging does not improve clinical outcomes. ${ }^{4}$ One RCT reported that among low-risk patients, spinal magnetic resonance imaging may increase the use of surgery, without improving average outcomes. ${ }^{5}$ Similarly, RCT evidence suggests that patients who receive imaging results may suffer from labeling effects: they report worse pain, perceive themselves to be less healthy, and seek more doctor visits than those who receive no imaging results. ${ }^{6,7}$ Observational studies also report longer work disability and increased use of subsequent diagnostic tests, spinal injections,

Published online September 28, 2015 and surgery among low-risk patients who receive early diagnostic imaging. ${ }^{8}$

Clinical guidelines have often suggested that older adults, such as those studied by Tan and colleagues, are a high-risk group who should undergo routine imaging. But a recent observational study suggests that even in this population, otherwise low-risk patients who receive early imaging (within 6 weeks of initiating a new episode of care) do not have improved outcomes, and have substantially increased rates of other clinical interventions. ${ }^{9}$

Despite guideline recommendations for parsimonious use, spine imaging rates increased rapidly during the early 2000s. Although we cannot make causal inferences, population-level clinical outcomes of back pain appeared to worsen during this period of rapid increase in spine imaging. ${ }^{10}$ Together, these lines of evidence suggest a plausible, though counterintuitive, hypothesis that spine imaging in low-risk situations more often leads to harm from irrelevant findings than to a benefit from discovering unsuspected disease. It may be that in this circumstance, sins of commission outweigh the sins of omission, and patients are more likely to suffer from having imaging than from not having it.

Tan and colleagues demonstrate that spine imaging rates remain higher than expected. The reasons are unclear, but are likely multifactorial, and may include fear of lawsuits, low tolerance for uncertainty, patient pressure, and unawareness or mistrust of guideline recommendations. Given the common bias for action among both patients and clinicians, many may perceive the consequences of missing an underlying systemic disease to be worse (and more easily identified) than the consequences of finding irrelevant conditions that lead to unnecessary care. Tan's finding of wide variation among clinicians, even among carefully selected low-risk patients, undermines the argument that "I see sicker or more complex patients than other clinicians." The variation may instead reflect different levels of clinician discomfort in the face of uncertainty, differences in counseling skills, or in patient expectations.

Patient pressure, sometimes in the form of satisfaction surveys, may have a particularly important role in this setting. Many patients assume that more care, especially "high-tech" care, can only be good. Clinicians who limit imaging may be perceived as not taking the pain seriously, preoccupied with 
cost-cutting, or hostile to patient autonomy. Patient-perceived need is strongly associated with the likelihood of spine imaging. ${ }^{11}$ Furthermore, in one RCT, patients who received radiographs were more satisfied with their care despite reporting worse pain and overall health. ${ }^{6}$ One survey suggested that many primary care physicians would order an MRI for an insistent patient in an obviously low-risk clinical scenario, even after explaining it was unnecessary. And claims data indicate that clinicians who face patient satisfaction incentives are more likely to use early imaging and more advanced imaging, even in low-risk patients. ${ }^{12}$

Given this panoply of challenges, what can be done to limit the use of unnecessary spine imaging? Tan and colleagues found that some clinicians are very parsimonious in the use of imaging, and perhaps we can learn from them some successful communication and reassurance strategies. I believe clinicians need better scripts for providing such education. An early RCT suggested that patient education strategies could at least maintain patient satisfaction in the absence of imaging. ${ }^{13}$ I often suggest that patient reassurance come after a physical examination, so that it does not ring hollow. Part of the message in the RCT was that imaging could be done later if circumstances change, and thus clinicians did not appear to be stonewalling.

The RCT also suggested that patients learn from what we do. Those who had imaging came to expect that it was appropriate care and should be done for anyone with back pain. ${ }^{13}$ Perhaps if we begin to lower imaging rates overall, the norm among patients, families, and friends will shift away from the expectation of routine imaging.

Other strategies for promoting more selective imaging also appear promising. Prior authorization requirements by insurance carriers appear effective, but onerous. Some have studied the use of computer-based ordering filters, requiring particular clinical findings before a spine image can be ordered, with radiologist consultation required for exceptions. A combination of education, audit, and feedback appears effective in some studies. Providing immediate therapeutic options such as same-day or next-day physical therapy may help to allay patient concerns about being taken seriously, as well as minimizing other interventions. A combination of all these efforts may actually be most promising.

Reducing repeat or follow-up imaging tests may also be an important strategy. Computer-based systems can offer reminders of previous spine imaging tests. Furthermore, reducing alarm at incidental findings on an initial imaging test may be important. A small observational study suggested that appending information to imaging reports regarding the prevalence of imaging findings in asymptomatic individuals may reduce the use of subsequent imaging (and some treatment interventions).
In a society that often assumes more is better, newer is better, more expensive is better, and that information can only be good, it is challenging to convey the message that sometimes less is more. Glib pronouncements to this effect are unlikely to be reassuring, and appeals to "evidence-based medicine" may be taken as a sign that something is being rationed. Efforts to promote more selective use of medical technology, such as the Choosing Wisely campaign, will succeed only if we can find better ways of communicating to the public, patients, clinicians, and trainees both the health and financial benefits of carefully targeted, parsimonious practice.

Corresponding Author: Richard A. Deyo, MD, MPH; , 3181 SW Sam Jackson Park Road, Mail Code FM, Portland, OR 97239, USA (e-mail: deyor@ohsu.edu).

\section{REFERENCES}

1. Chou R, Gaseem A, Owens DK, Shekelle P. Diagnostic imaging for low back pain: advice for high-value health care from the American College of Physicians. Ann Intern Med. 2011;154(3):181-189.

2. Tan A, Zhou J, Kuo Y-F, Goodwin JS. Variation among primary care physicians in the use of imaging for older patients with acute low back pain. J Gen Intern Med. 2015. doi:10.1007/s11606-015-3475-3.

3. Brinjikji W, Luetmer PH, Comstock MS, Bresnahan BW, Chen LE, Deyo RA, Halabi S, Turner J, Avins AL, James K, Wald JT, Kallmes DF, Jarvik JG. Systematic literature review of imaging features of spinal degeneration in asymptomatic populations. Am J Neuroradiol. 2015;36:811-816.

4. Chou R, Fu R, Carrino JA, Deyo RA. Imaging strategies for low-back pain: systematic review and meta-analysis. Lancet. 2009;373:463-472.

5. Jarvik JG, Hollingworth W, Martin B, et al. Rapid magnetic resonance imaging vs radiographs for patients with low back pain: a randomized controlled trial. JAMA. 2003;289:2810-8.

6. Kendrick D, Fielding $\mathbf{K}$, Bentley E, Kerslake R, Miller P, Pringle $\mathbf{M}$. Radiography of the lumbar spine in primary care patients with low back pain: randomised controlled trial. BMJ. 2001;322(7283):400-405.

7. Ash LM, Modic MT, Obuchowski NA, Ross JS, Brant-Zawadzki MN, Grooff PH. Effects of diagnostic information, per se, on patient outcomes in acute radiculopathy and low back pain. AJNR Am J Neuroradiol. 2008;29:1098-103.

8. Webster BS, Choi YS, Bauer AZ, Cifuentes M, Pransky G. The cascade of medical services and associated longitudinal costs due to nonadherent magntic resonance imaging for low back pain. Spine. 2014;39:1433-40.

9. Jarvik J, Gold LS, Comstock BA, Heagerty PJ, Rundell SD, Turner JA, Avins AL, Bauer Z, Bresnahan BW, Friedly JL, James K, Kessler, Nedeljkovic SS, Nerenz DR, Shi X, Sullivan SD, Chan L, Schwalb JM, Deyo RA. Association of early imaging for back pain with clinical outcomes in older adults. JAMA. 2015;313:1143-53.

10. Deyo RA, Mirza SK, Turner JA, Martin BI. Overtreating chronic back pain: time to back off? J Am Board Fam Med. 2009;22:62-8.

11. Wilson IB, Dukes K, Greenfield S, Kaplan S, Hillman B. Patients' role in the use of radiology testing for common office practice complaints. Arch Intern Med. 2001;161:256-263.

12. Pham HH, Landon BE, Reschovsky JD, Wu B, Schrag D. Rapidity and modality of imaging for acute low back pain in elderly patients. Arch Intern Med. 2009;169(10):972-981.

13. Deyo RA, Diehl AK, Rosenthal M. Reducing roentgenography use. Can patient expectations be altered? Arch Intern Med. 1987;147:141-5. 